\title{
Pipelined Soft Packaging Cargoes Handling Robot
}

\author{
Li guangyao, Fu Xinzhong, Du Yunjia and Xu Shuqionga \\ School of Mechanical and Vehicle Engineering, Lin Yi University, Shandong, China
}

\begin{abstract}
In order to solve the problem of packing and packing on the soft package in the pipeline,we use PLC system to control the robot. The step motor and the push rod motor cooperate with each other to $r$ ealize the space movement. The vacuum sucker can firmly absorb the target and realize the transportation a nd packing of the target. To solve the problem of heavy workload of the soft packing line, wasting the manp ower and material resources, we change the past hard grab transport mode and use the vacuum sucker to abs orb the soft package to improve the packing efficiency. As for transporting soft package goods, the vacuum sucker has characteristics of small wear, fast speed, light and convenience. PLC control system greatly impr oves the accuracy of its moving objects, the robot is more efficient and practical.
\end{abstract}

\section{Development background and significance}

The mass production of soft packaging goods industry makes the transportation packing of products become a major problem. Nowadays, with the rapid development of industrialization, palletizing robot has become the most convenient choice [1]. Nevertheless, robot loading is also facing many problems. If we want to pack it at a higher speed, it will be more difficult. At present, most of the soft packaging goods on the assembly line (such as milk, washing liquid, salt and so on) are mostly used by manual or automatic manipulator to carry the packing. Because the packaging material is relatively soft, the mechanical mobile phone manipulator is easy to slide and easy to break, and the manual transportation is inefficient, time-consuming and laborious.

To solve the above problems, we have designed an automated pipeline handling robot, mainly by changing the manipulator to a double-layer vacuum sucker device. When working, the stepper motor and the push rod motor are positioned, the air compressor works, the suction cup is crawled and the soft package is put into the box. The device realizes the cooperation of all parts by program control.

\section{Main functions and performance indicators}

Replacing the mechanical hand with an industrial vacuum sucker can greatly improve the transport efficiency of the soft packaged goods. At present, the type of palletizing robots,such as the four-axis palletizing robot, the six-axis palletizing robot, etc., are all used for palletizing and packing products like human arms, for the end of the production line. Bagging, boxed, barreled and other articles are stacked from production line to pallet. They are mainly used in feed, fertilizer, chemical industry, food, beverage, cereals and oils industry[2]. However, due to the incompleteness of the control system, the traditional manipulator has poor antiinterference ability and low accuracy. Especially for soft commodities, if the traditional manipulator catch, the slightest mistake will damage the moving objects[3]. The PLC control system is the core of detection and control. The infrared photoelectric sensor is used to detect the moving objects. The photoelectric sensor or micro switch is used to determine whether the sucker is in place. Through the step motor and the synchronous belt, the robot moves in the horizontal plane of the $\mathrm{X}$ and $\mathrm{Y}$ axis. Through the push rod motor, the motion of the $\mathrm{Z}$ axis is realized. Therefore, the robot has the advantages of convenient control and flexible execution.

Technical index (take milk as an example): fill a box of soft bags, 24 bags of milk only need 30 seconds, an operator is enough to operte the machine Compared with the traditional mechanical mobile phone users, this machine can save time and ensure the integrity of goods. It greatly saves manpower and material resources.

\section{Innovation and application}

(1) Change the past soft packing goods by the way of manipulator packing, use the vacuum sucker to absorb the goods, and improve the packing efficiency. The vacuum sucker is small, fast, light and convenient for transporting soft package goods.

(2) The PLC control system is adopted in the control system[4], which greatly improves the accuracy of the moving objects and makes the handling of the soft bags greatly improved.

(3) The vacuum sucker robot works more smoothly, reduces noise pollution, and is more efficient and practical. 
(4)The characteristic of this project is to solve the problem of heavy workload and waste of manpower and material resources in the transportation of goods in the flexible packaging line. Changing the traditional palletizing robot's hard grasp and transportation mode, using vacuum sucker to absorb soft bags and sucker transport is a major feature of this project.

Market and benefit analysis and application prospect forecast: in soft handling of goods such as soft, liquid detergent. Salt and other applications are widely used. Nowadays, people's living standard is increasing, and the demand for soft package and washing liquid is increasing, so more and more enterprises have been sprouted. Therefore, the handling of soft packaged goods is becoming more and more important. Therefore, the automatic pipelining machine we designed for this kind of handling is relative to the traditional machine. Armed hands are more advantageous and the market demand is huge[5].

The machine is mainly composed of two stepper motors, PLC control panel, push rod motor, solenoid valve, vacuum generator and air compressor(as shown in FIG. 1).

The device provides the air compressor for the pressure $5 \mathrm{kPa}$ air compressor, through the vacuum generator to the suction grab soft package, through the $X$, $\mathrm{y}, \mathrm{Z}$ three axis coordination motion to complete the packing of the soft package(as shown in FIG. 2)[6].

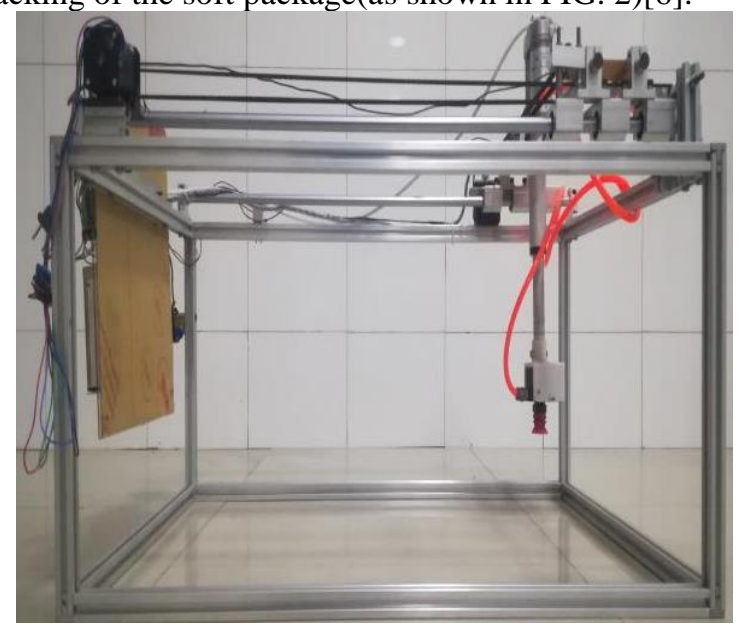

Figure 1. Pipelined soft packaging cargoes handling robot model

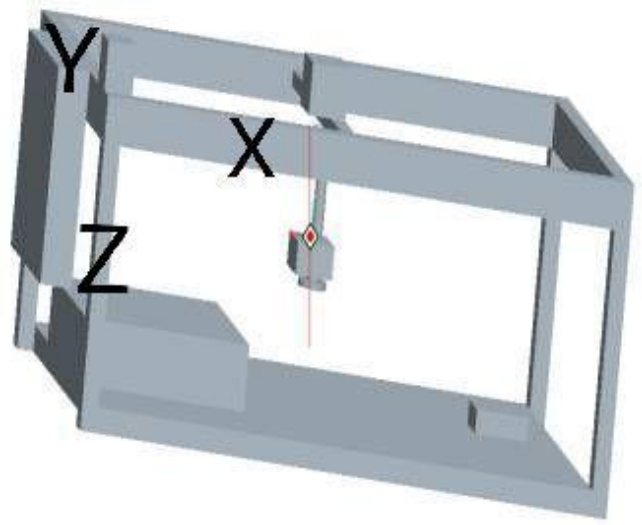

Figure 2. Robot PROE model

\section{Design scheme}

\subsection{Main module of soft packaging cargo handling robot}

PLC control system: as the brain of a robot, it controls the precise and orderly work of various institutions, reduces the work error, and controls the location more accurately.

Space moving mechanism: the three axes of $X$, $y$ and $\mathrm{Z}$ are composed of optical axis guide. The motor is controlled by the control system, and the three axis synchronous motion can be carried out to shorten the handling time.

Target grabbing mechanism: its function is to absorb the target. It is composed of air compressor, vacuum generator and vacuum sucker. It can firmly adsorb the target and then carry out the handling with the space mobile mechanism.

\subsection{The design of the frame.}

Using square aluminum and optical axis guide to build a two-dimensional platform, for the $\mathrm{X}$ axis, the step motor drives a drive shaft to drive the slider, making the device driven by the $\mathrm{X}$ axis equal, reducing the torque generated by the deformation and friction, the push rod motor and the vacuum generator on the $\mathrm{Y}$ axis, because the force arm is shorter and the torque is smaller. It can be ignored, so only one synchronous belt is used to reduce the processing and consumables. The $\mathrm{Z}$ axis is driven by the push rod motor to complete the clamping and placing of the soft bag. The maximum $X$ axis of the stroke can reach $150 \mathrm{~cm}$, the $Y$ axis can reach $100 \mathrm{~cm}$, and the $Z$ axis can expand to $20 \mathrm{~cm}$.[7]

\subsection{A brief description of the function of each part.}

In Figure 3, number 1 for a push rod motor, its main function is to drive the telescopic sucker up and down movement, to achieve soft grasp;

The number 2 is the vacuum generator, and the suction plate is used to adsorb the soft package. The characteristic is that the gas volume is small, the vacuum degree is not high, and it can work intermittently (as shown in FIG. 4).

The number 3 is grasping soft silicone sucker,with special materials and soft, good air tightness, can be firmly adsorbed roolls (as shown in FIG. 5);

The number 4 is stepping motor,can precise positioning, and minimize the error.

The number 5 is the emergency stop button. In case of emergency, press the button to force the shutdown.

The number 6 is represented as a PLC control system. Using PLC as the core of the system, the performance is more stable and the location is more accurate. For example, the default packing value is 8 packets, according to clockwise direction, first, put one layer, and then the second layer. For the box position and the small shift of the position of the soft packet, the laser 
positioning can adjust it. For the exceeded scope of the machine activity, it can be restarted by mounting a limited switch, stopping automatically when it reaches the maximum stroke and straightening by operators.

The number 7 and 8 are the soft bag packaging box respectively.

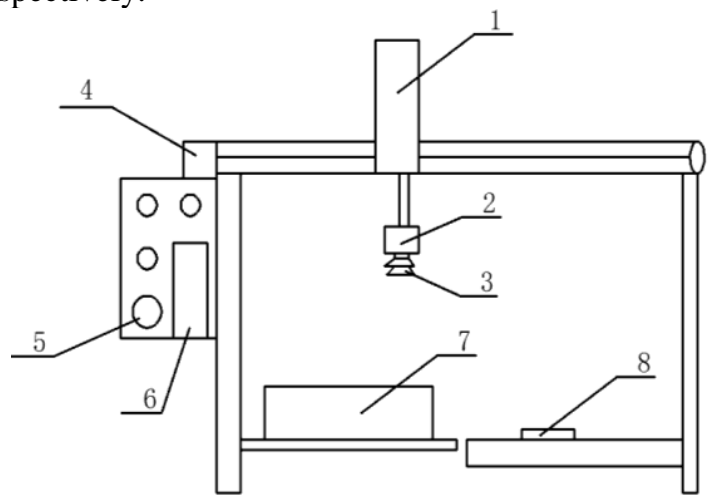

Figure 3. Frame diagram of a handling robot.

1.Push rod motor 2.Vacuum generator 3. Soft sucker 4. Stepper motor5.Urgent stop button 6. PLC control system 7.Commodity box 8.Soft packaged go

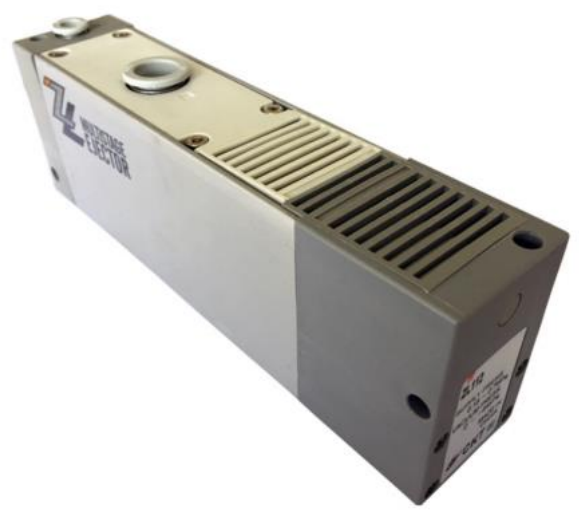

Figure 4. Vacuum generator

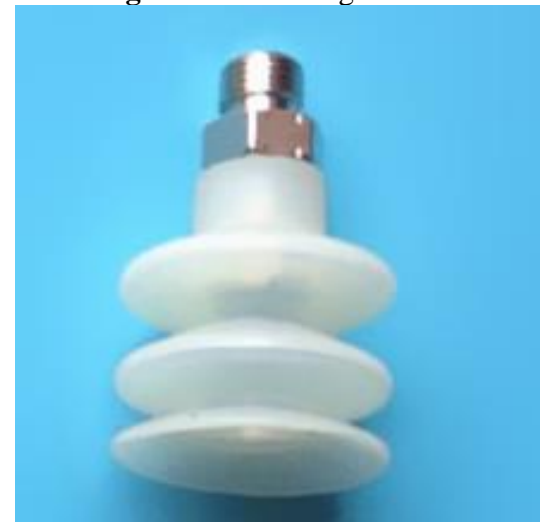

Figure 5. Soft sucker

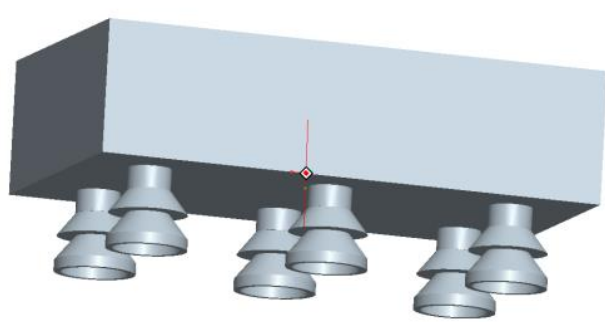

Figure 6.Vacuum sucker simulator

\subsection{Program designs}

(1) First, determine the $X, y$ and $Z$ axes. Two stepper motors respectively control $\mathrm{X}$ and $\mathrm{Y}$ axis, and push rod motor controls $\mathrm{Z}$ axis.

(2)Machine does edge scanning, calibrate position and determine the position of the soft bag;

(3) Drive the motor which design the $x, y, z$ axis at the same time to the soft top position;

(4) The program controls relay, and then the vacuum generator works. When the pressure valve opens, it will suck the soft bag up;

(5) Packing after sucking the bag up;

(6) Repeat this way. After each trip, the program automatically adjusts the homing and minimes the error.[8]-[10]

\subsection{Instructions for the use of this machine}

Place the machine in the packing position and open the machine, the machine will primarily calibrate the position. It will go back to the grab area of the soft bag. Moving the machine and making it aim at the position of the soft package can make it easy to quickly grab the soft bag during the next packing process. The calibration only needs to calibrate it once when fixed, and the machine is directly started in the subsequent use. It can run normally.

The emergency stop button is a button designed specially for the machine to break down, and when it fails, it can press the stop button directly[11].

\section{Conclusions}

Our design solves the problem of the soft packing and packing in the current pipeline. Through the step motor and the push rod motor, the space movement is realized. The vacuum sucker is used to draw the target firmly. The PLC control system is adopted to greatly improve the precision of the handling material, which is more efficient and more practical.

\section{References}

1. Shi Shengzhi, Wei Yili, Han Suxian. Application Research of packaging palletizing automatic production line. Guide to scientific and technological innovation, 2009, (22): 72

2. Hu Hongguo, Gao Jianhua.A review of palletizing Technology.Combined machine tool and automatic machining technology.2000(6):7-9.

3. Li Jinquan, Yang Xiangdong,Fu Tie.Structure and control system structure of palletizing robot.Beijing Institute of Technology.2011.

4. Shi Shouyong, SIEMENS S7-200 SMART PLC programming and Application Tutorial, 2016.8.

5. Yue Manlin, Jiang Jihong.Design of automatic palletizing packaging production line control system.Mechanical Engineer.2007(2):80-81. 
6. Chen Baojiang, Ge Tianzi, Wang Jianzhi.Research on a kind of Packaging Manipulator and its control.Packaging engineering.2014,35(7):90-94.

7. Dong Jing, Jiang Yongzong.Design of a palletizing control unit for automatic fertilizer packing palletizing machine. Journal of agricultural mechanization research.2014,36(3).

8. Lu Zhizhen. Design of control system for automatic packaging palletizing production line based on PLC.Jiangnan University.2008.

9. Liao changchu. PLC programming and Application.Machinery Industry Press, Beijing.

10. Li Xuemei, Zeng Dehuai.Design and application of vacuum sucker.Hydraulic and pneumatic.2004(3).

11. Z.Y.Rui,T.Liu.Motionand Simulation of palletizing robot.Mechanical manufacture. 2010,48(548):7-10. 